\title{
CT of amebic liver abscess: different morphological types with different clinical features
}

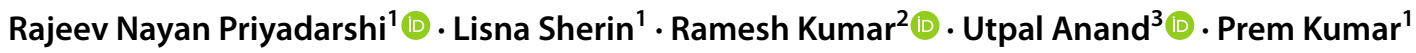

Received: 28 February 2021 / Revised: 8 April 2021 / Accepted: 10 April 2021 / Published online: 24 April 2021

(c) The Author(s), under exclusive licence to Springer Science+Business Media, LLC, part of Springer Nature 2021

\begin{abstract}
Purpose To identify different morphological types of amebic liver abscess (ALA) based on CT findings and to assess whether they have different clinical features.

Method CT images of 112 symptomatic patients with ALA were analyzed to identify the imaging features distinctive of each morphological type. The following CT findings were investigated: the presence of abscess wall, rim enhancement, edge characteristic, septa, intermediate density zone, and peripheral hypodensity. Abscesses from each type were further evaluated for their clinical presentations, laboratory findings and outcomes.

Results We identified three types of ALAs: type I, II and III. Type I abscesses (66\%) were characterized by absent or incomplete walls, ragged edges and peripheral septa; their edges exhibited irregular and interrupted enhancement. Type II (28\%) had a complete wall characterized by rim enhancement and peripheral hypodense halo. Type III (6\%) demonstrated a wall but without enhancement. Clinically, type I abscesses presented acutely with severe disease. They had significantly deranged laboratory parameters, higher incidence of rupture and higher rate of inpatient or intensive care unit admission. The severity of the disease prompted immediate percutaneous drainage in most type I abscesses (81\%). Two of them died from multiple organ failure. The type II or III abscesses, on the other hand, had delayed presentations with mild to moderate disease, with near normal laboratory findings.

Conclusion ALAs have three different CT morphological types, with different clinical and laboratory features. Percutaneous drainage is indicated in most of type I abscesses.
\end{abstract}

Keywords Severe liver abscess · Abscess classification - Abscess rupture $\cdot$ Rim enhancement $\cdot$ Amebic colitis · Percutaneous drainage

Rajeev Nayan Priyadarshi

drrajeevnp@gmail.com

Lisna Sherin

lisnasherinm@gmail.com

Ramesh Kumar

docrameshkr@gmail.com

Utpal Anand

utpalanand2@gmail.com

Prem Kumar

drprempat@gmail.com

1 Department of Radiodiagnosis, All India Institute of Medical Sciences, Patna, Bihar, India

2 Department of Gastroenterology, All India Institute of Medical Sciences, Patna, Bihar, India

3 Department of Surgical Gastroenterology, All India Institute of Medical Sciences, Patna, Bihar, India

\section{Introduction}

Amebic liver abscess (ALA), a parasitic infection caused by protozoan Entamoeba histolytica, is the commonest cause of liver abscess in developing countries [1-3]. It occurs as an extraintestinal manifestation of intestinal amebiasis. Most ALAs present with mild to moderate symptoms and respond within 3 days of antiamebic therapy [4]. However, reports from endemic areas indicate that the disease can be severe - not controlled with the drug alone-in up to $40 \%$ of patients. Such abscesses often present acutely with rupture, organ failure and deranged laboratory findings; they require additional percutaneous drainage [2, 3, 5-7]. High incidence of malnutrition and alcohol consumption are risk factors for the increased severity in endemic areas $[6,8]$.

The characteristic CT appearance of ALAs is considered non-specific and has been described as a round or oval 
hypodense lesion with a thick enhancing wall and peripheral edema [9-12]. Although considered to be classical, these features are suggestive of resolving ALAs. Studies have recognized that wall formation and rim enhancement are late findings identified while the healing process proceeds $[13,14]$. This appearance therefore tends to reflect mild drug responsive disease. In our experience, the abscesses that present acutely rarely exhibit these features. Clinically, such abscesses are associated with severe symptoms or with deranged laboratory profile. It is therefore important to distinguish such aggressive abscesses from those with mild symptoms.

Imaging morphology of ALAs is known to vary considerably depending on the effect of treatment and time of presentation [13-16]. Two studies (both in non-English literature)) have indicated that ALAs can be classified into several morphological types according to their sonographic features; the classification was useful in identifying the abscesses that should be considered for percutaneous drainage [17, 18]. No study, however, has considered that different morphological types could be related to varying clinical and laboratory features. This understanding can add much value for radiologists, physicians and surgeons.

The aim of this study is twofold: first, to identify different morphological types based on CT findings, and second, to assess whether different types have different clinical features and outcomes.

\section{Method}

\section{Patient population}

CT examinations $(n=167)$ retrieved from the hospital PACS of 147 consequent patients with ALA treated between April 2018 and December 2020 were retrospectively reviewed. The diagnosis of ALA was established by serological test, imaging features, and characteristics of the aspirated pus. Of 147 patients, 35 were excluded: 10 because the aspirate was culture positive, 11 because they had prior percutaneous intervention, 6 because they had only non-contrast CT due to renal dysfunction, and 8 because their abscesses were small $(<5 \mathrm{~cm})$ for morphological characterization. A total of $132 \mathrm{CT}$ examinations from 112 patients were identified. After excluding 20 follow-up studies, 112 examinations were considered for final analysis. This study also included 43 patients from our previous study on venous thrombosis in ALA; this complication was not analyzed in this study [19]. Permission from the Institute Ethics Committee was obtained (AIIMS/Pat/IRC/2020/PGTh/July 19/15).

All patients were native residents of Bihar (eastern India). Most patients in this study were adult (mean $43.2 \pm 12$ years), with only three patients $<18$ years of age.
108 patients $(96 \%)$ were men. Nearly all had rural backgrounds, and $87 \%$ had a history of alcohol (mostly prepared from palm sap) drinking. None of the patients had coexisting comorbidity, but a 4-year child was found to be a novel coronavirus (COVID-19) positive with coexisting pneumonic consolidation in the left lung. All patients had received antiamebic therapy for at least one day before CT examination, but only 52 patients $(46 \%)$ had completed a 7-day course of antiamebic drug (metronidazole). All patients were symptomatic at the time of initial CT.

\section{Morphological analysis}

All CT examinations were performed on a dual-source 256 slice CT system (Siemens SOMATOM Definition Flash; Siemens, Germany). Portal venous or hepatic venous images (acquired at 60 and $120 \mathrm{~s}$ after about $80-100 \mathrm{~mL}$ of iohexol injection, respectively) and non-contrast images were reviewed. The $\mathrm{CT}$ images of $0.6 \mathrm{~mm}$ slice thickness were evaluated on workstations. We attempted to classify the abscesses into different morphological types based on the following characteristics: (1) presence of wall, (2) rim enhancement, (3) characteristics of the abscess edge, (4) presence of the septa, (5) presence of intermediate density zone on non-contrast CT, and (6) presence of peripheral hypodensity. The largest abscess was considered for the analysis in patients with multiple abscesses. The sonographic images were also reviewed in conjunction with the CT images, particularly for the presence of solid necrotic tissue. Three radiologists, blinded to clinical findings, independently reviewed the images. Decisions about types of abscess morphology and other imaging characteristics were reached by consensus.

To assess whether different morphological types might have different clinical features, we reviewed the clinical symptoms and laboratory data at the time of CT examination. The laboratory data included total leukocyte count, albumin, total bilirubin, alanine aminotransferase, aspartate aminotransferase, and alkaline phosphatase. Also, the time interval between the onset of symptoms and CT examinations were recorded. In addition to these clinical data, to investigate the differences in disease severity among different types, we further evaluated the imaging findings that are known to be associated with severe disease [20]. The additional CT findings recorded were as follows: (1) size, (2) number of abscesses, (3) presence of rupture, (4) presence of hemorrhage or gas-bubbles, (5) presence of intrahepatic duct dilation, (6) colonic thickening, (7) presence of ascites, and (8) presence of pleural effusion. Also, we assessed how the different morphological types responded to antiamebic therapy. 


\section{Treatment received}

All patients were intended to be treated with drug therapy alone. Percutaneous ultrasound-guided drainage was performed for unresponsive or poorly responsive abscesses as defined by the clinical criteria: (1) abscesses with severe clinical signs, not controlled by drugs, (2) deranged laboratory parameters, (3) abscesses with rupture. 83 of 112 patients $(74 \%)$ patients required drainage: 59 percutaneous catheter drainage, 24 only needle aspiration. All drainage procedures were performed within 4-days of imaging study. One patient with cecal perforation required surgery.

\section{Definitions}

The abscess wall was defined as complete when a welldefined rim of tissue surrounded the entire circumference of the cavity. Its presence was indicated by a uniform rim enhancement. The rim enhancement was defined as a complete ring-like enhancement of the abscess wall. The abscess edge was considered ragged when the irregular boundary of abscess was formed directly by the liver tissue in absence of a well-developed wall. The septa were defined as thin irregular projections into the cavity from the abscess margin, with a height $\geq 5 \mathrm{~mm}$. The perilesional hypodensity was considered halo-like when it was limited around the abscess wall, forming a halo. The intermediate density zone was defined as an intermediate attenuation zone on noncontrast CT image between the central liquefied necrotic tissue and peripheral healthy tissue. The solid necrotic tissue was defined as tissue that was detected on sonography, but not on contrast-enhanced CT.

\section{Data analysis}

To examine the differences in each variable between the different types of ALA, we used one-way analysis of variance and the Chi-square test or the Fisher exact test. Data were analyzed using SPSS software (version 22.0). A $p$-value less than 0.05 was considered significant.

\section{Results}

\section{Morphological types}

On the basis of the CT morphology, we were able to classify all abscesses into three types: type I, type II and type III. They were identified on the basis of four specific CT features as listed in Table 1. Six abscesses had overlapping or indistinct CT features, not fitting precisely into a particular morphological type. Assignment of these cases into a single morphological type was based on the combination of
Table 1 Distinguishing CT features of the three morphological types of amebic liver abscesses

\begin{tabular}{llll}
\hline CT features & Type I & Type II & Type III \\
\hline Wall & Absent or incomplete & Present & Present \\
Edge appearance & Ragged & Smooth & Smooth \\
Enhancement pattern & Absent or interrupted & Rim & Absent \\
Septa & Present & Absent & Absent \\
\hline
\end{tabular}

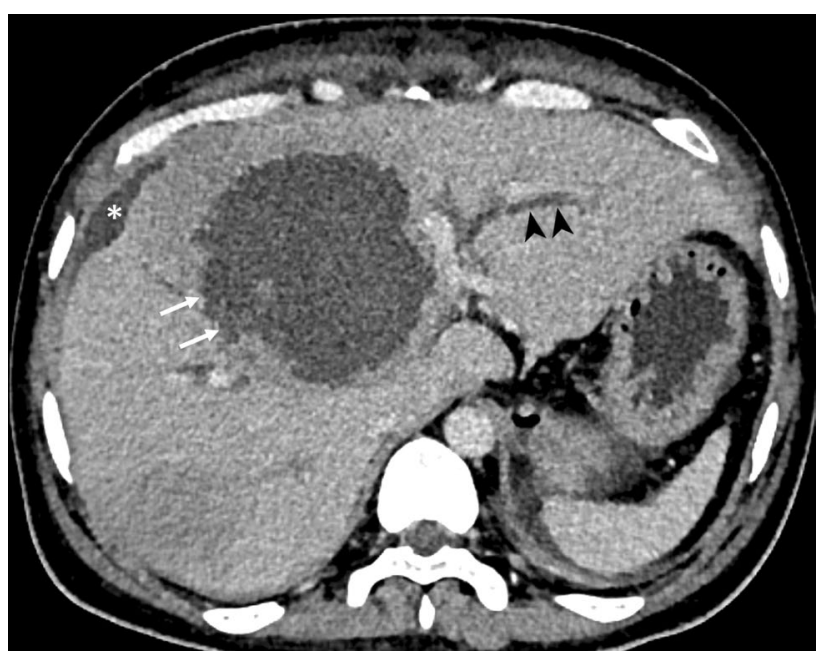

Fig. 1 CT of a 40-year-old man with a type I abscess. The axial CT image illustrates the non-enhancing and ragged edge of the abscess in the absence of a definite wall (arrows). Note the dilated intrahepatic biliary ducts (arrowheads) resulting from the mass effect. Also, note the localized perihepatic fluid collection from the abscess rupture (asterisk)

the CT and sonographic findings. The details of each type are as follows:

\section{Type I: ALA with ragged edge ( $n=74,66 \%$ cases)}

Type I abscesses had absent or incomplete walls; their edges were ragged. Of the 74 abscesses, 24 (32\%) displayed no contrast enhancement at the edges. The remaining $68 \%$ showed irregular and interrupted enhancement (Figs. 1, 2, 3, 4 and 5). Multiple irregular septa were observed in all cases. The septa were typically multiple, with size varying from $5 \mathrm{~mm}$ to $5 \mathrm{~cm}$. Collectively, these features resulted in a ragged-edge appearance to the abscesses (Fig. 5). All abscesses-despite the presence of septa-were unilocular because the septa never divided the abscess cavities. 51 abscesses (69\%) showed a diffuse or wedge-shaped hypodensity (Figs. 3, 4, 5 and 6). Five abscesses showed a transitional zone of intermediate density on non-contrast CT images (Fig. 7). On sonography, the type I abscesses had indistinct walls and appeared heterogeneous due to the 


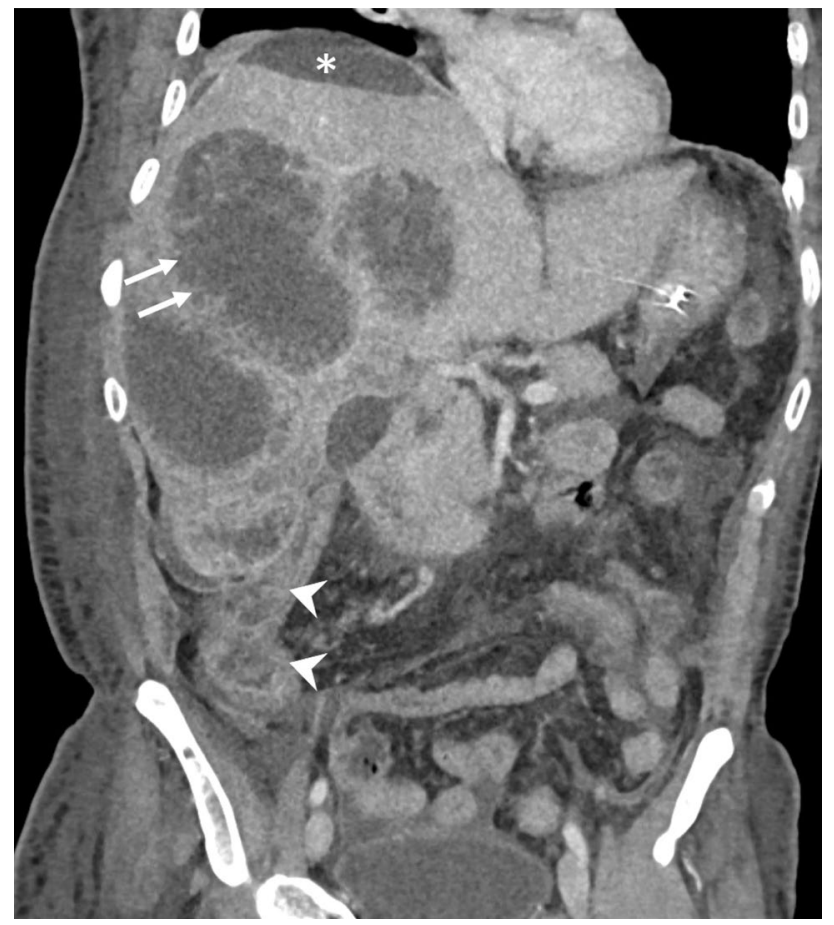

Fig. 2 CT of a 53-year-old man demonstrating the ragged and nonenhancing edges of multiple type I abscesses combined with the absence of their walls (arrows). Note the amebic colitis involving the cecum and ascending colon (arrowheads). The localized perihepatic fluid collection was due to rupture of one of the abscesses (asterisk). The patient presented in the emergency department with a shock-like state

presence of solid necrotic tissue. Three abscesses were misdiagnosed initially on sonography as malignant lesions due to heterogeneity caused by the solid material (Fig. 6a).

\section{Type II: ALA with complete rim enhancing wall ( $n=31,28 \%$ cases)}

Type II abscesses had a complete wall characterized by rim enhancement and relatively smooth outline. In 25 abscesses $(81 \%)$, a peripheral halo-like hypodensity was observed to form a double-target appearance (Fig. 8). The typical septa as seen in type I were lacking, but focal nodularity of the wall representing partially resorbed or remodeled septa was noted in several instances (Fig. 9). The cavities were comparatively more spherical than type I. On sonography, the proportion of solid necrotic material was reduced compared with type I abscesses (Fig. 8b).

\section{Type III abscess: ALA with non-enhancing wall $(n=7$, $6 \%$ cases)}

Type III abscesses, like the type II, also demonstrated a complete wall. However, the wall was distinct in that it

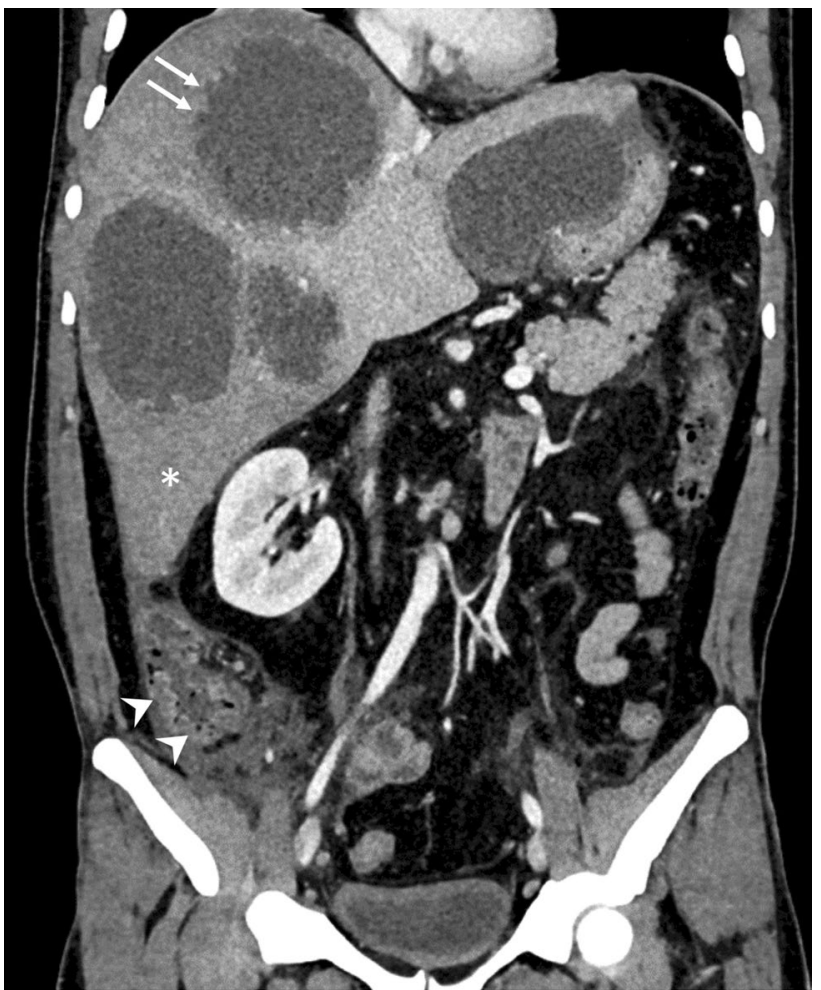

Fig. 3 Coronal CT image of multiple type I abscesses in a 45 -yearold man. The abscess walls are indistinct, and the edges are ragged and non-enhancing (arrows). There is a diffuse hypodensity around the abscesses (asterisk). Note the thickened cecal wall (amebic typhlitis) denoted by arrowheads. The localized fluid collection around the left lobe of the liver resulted from the abscess rupture

was much smoother and did not enhance with contrast. A faint ill-defined peripheral hypodensity was observed in 4 abscesses; it helped to distinguish the type III abscesses from other cystic lesions, such as hydatid or simple hepatic cysts (Fig. 10). The septa were not observed in any cases. On sonography, minimal or no solid component was observed, and the content was more homogenous than type II.

\section{Additional significant CT Findings}

Overall, most abscesses were round or oval; however, they assumed variable shapes with polycyclic or lobulated borders when multiple lesions coalesced together (Figs. 4, 10, 11). Type I abscesses were frequently multiple (mean 2.4 vs 1.4 vs $1.1, p=0.039$ ) compared to type II or type III abscesses. Ascites and pleural effusion were more frequently observed in patients with type I abscess. Gas-bubbles, hemorrhage, and intrahepatic duct dilatation were almost exclusively observed in type I abscesses (Figs. 1 and 11). Table 2 summarizes the additional CT findings, clinical features, laboratory results and treatment outcomes of different types of abscesses. 


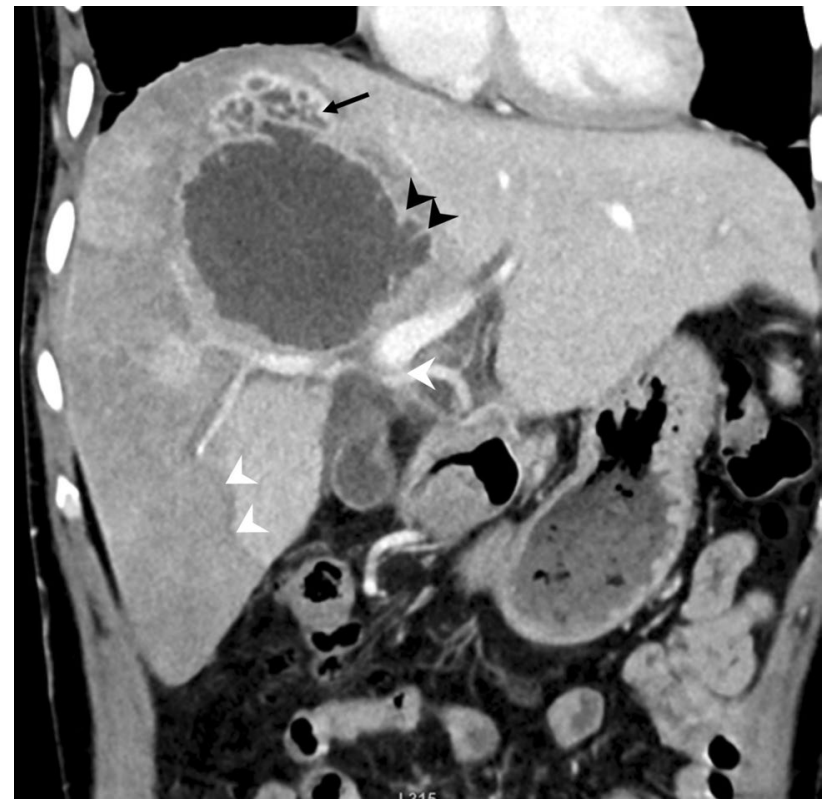

Fig. 4 A 35-year-old man with a type I abscess showing incomplete wall, multiple septa (black arrowheads), irregular edges, interrupted contrast enhancement, and wedge-shaped hypodensity in the right lobe (white arrowheads). Note the lobulated outline of the abscess superiorly (arrow) resulting from the coalescence of smaller abscesses

Rupture occurred in 44 (39\%) of 112 cases (39 intraperitoneal and 5 intrathoracic), far more frequently in the type I than type II or type III abscesses (57\% vs $6 \%$ vs $0 \%$, $\mathrm{p}<0.001)$. Notably, the ruptures had already occurred before the presentations in 40 (95\%) of 42 ruptured type I abscesses. The fluid collections resulting from intraperitoneal rupture were localized in the perihepatic area in 32 cases (Figs. 1, 2, 3, and 7). In the remaining 7 cases, they diffusely involved the entire peritoneal compartment (Fig. 11).

Concurrent colonic involvement was observed in 32 (28\%) of 112 cases, more frequently in patients with type I than type II or type III abscesses (38\% vs $13 \%$ vs $0 \%$, $p=0.008$ ). Cecum was affected (amebic typhlitis) in all patients (Fig. 3). The appendix was contiguously involved (typhloappendicitis) in 12 patients. The ascending colon was involved in five patients; the transverse colon, in two patients (Fig. 12). Symmetric wall thickening, similar to inflammatory bowel disease, was the most common CT finding. Colonic perforations occurred exclusively in type I abscesses. The appendicular and cecal perforations were observed in three and two patients, respectively; all had a localized fluid collection in the right iliac fossa or pelvis (Fig. 13). Ameboma (a mass-like thickening of the cecum caused by ameba) was observed in two patients.

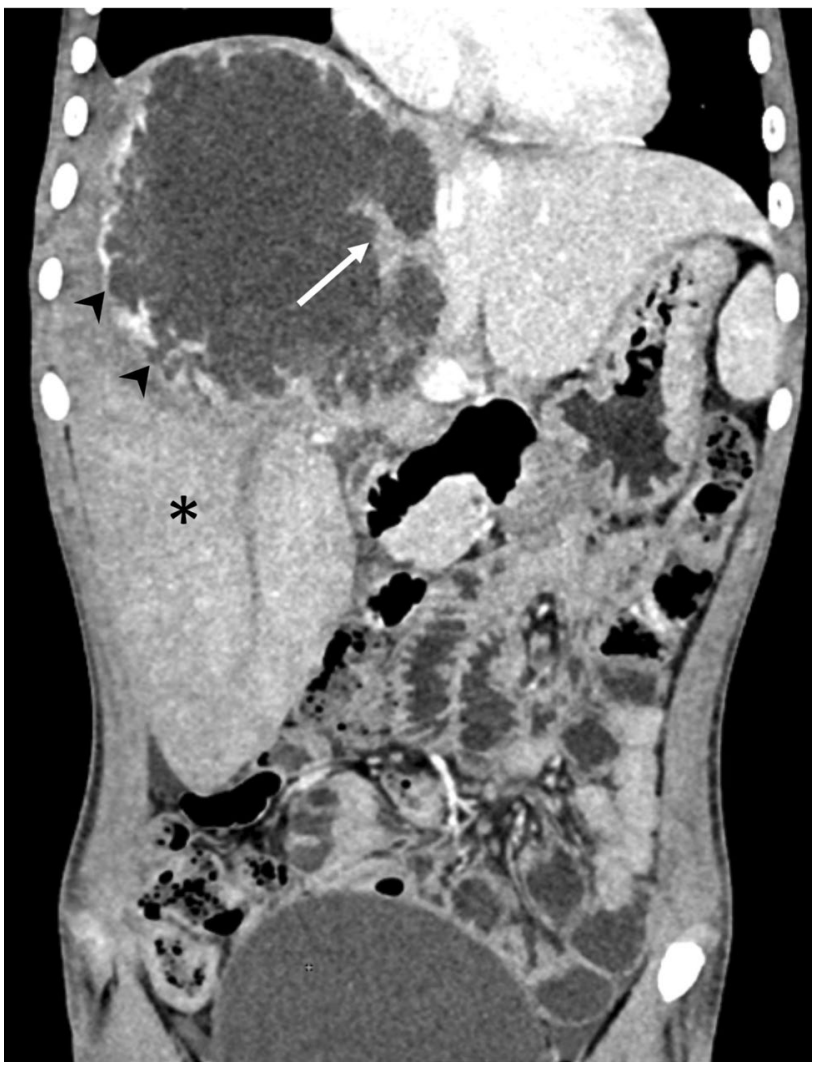

Fig. 5 Coronal CT image of a type I abscess in a 13-year-old boy featuring the ragged-edge appearance of the abscess formed by the presence of multiple septa, incomplete wall and interrupted enhancement of the edges (arrowheads). Also, note the presence of a diffuse hypodensity in the right lobe of the liver (asterisk)

\section{Clinical features, treatment and outcomes}

Striking differences in the clinical and laboratory findings were observed, corresponding to the types of abscesses. Most patients with type I abscesses presented earlier than those with type II or III (mean, 10.5 vs 27.6 vs 41.5 days, $p<0.001)$. As shown in Table 2, clinical symptoms, including fever, right upper quadrant pain or tenderness, were more severe in the patients with type I abscesses than other types. Peritonitis (resulting from abscess rupture) or septic shock-like state (even without pyogenic infection) was commonly seen in the patients with type I abscesses. Total leucocyte count, hepatic transaminases and bilirubin were significantly elevated, whereas serum albumin was lower in the patients with type I abscesses compared with other types. Of 74 patients with type I abscesses, 60 required drainage; the severity of the disease prompted immediate drainage in 52 patients before they could complete the drug therapy. 67 patients in this group required admission to the hospital and 15 of them required 


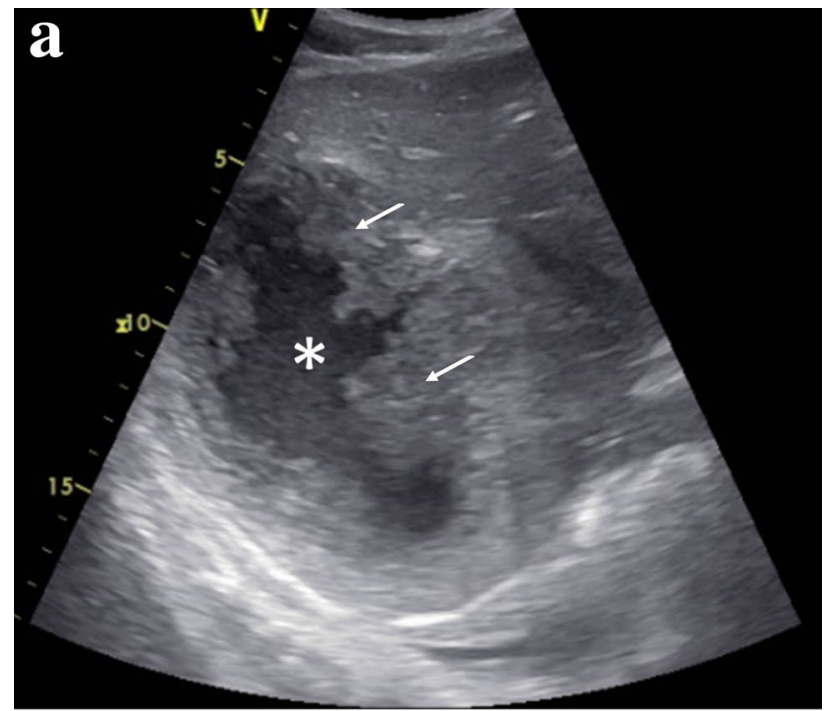

b

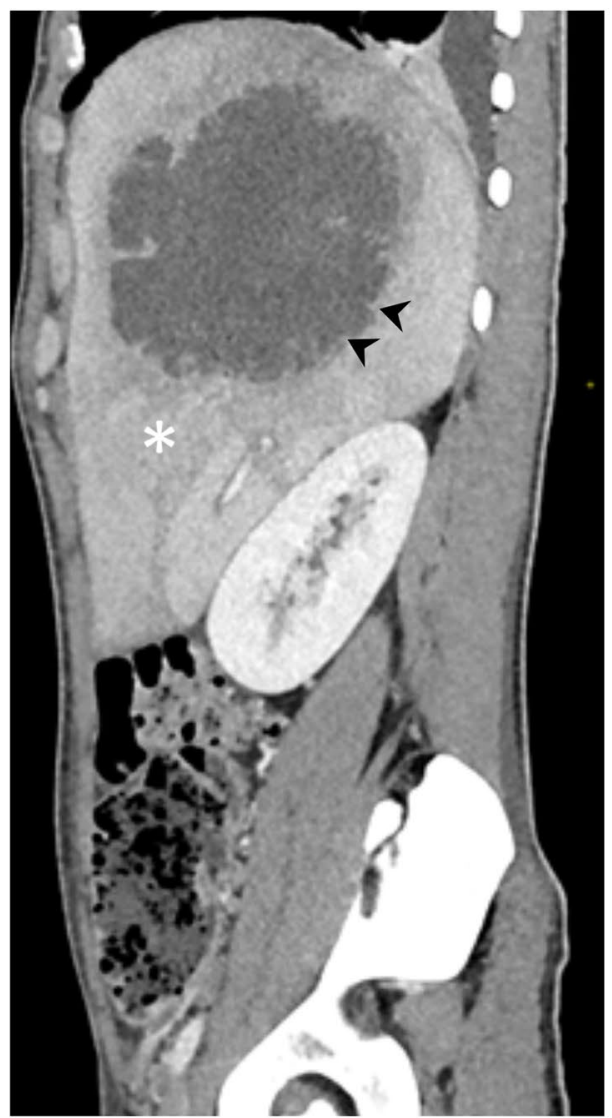

Fig. 6 a Sonographic image of the liver in a 42-year-old man showing a necrotic mass with central liquefied (asterisk) and peripheral solid material (arrows). Note the indistinct margin of the mass. The lesion was initially diagnosed as a malignant lesion on the basis of the sonographic findings, but CECT findings confirmed the type I pattern of the abscess. b Sagittal CT image shows the ragged appearance of the abscess edge (arrowheads) and the wedge-shaped hypodensity surrounding the abscess (asterisk)

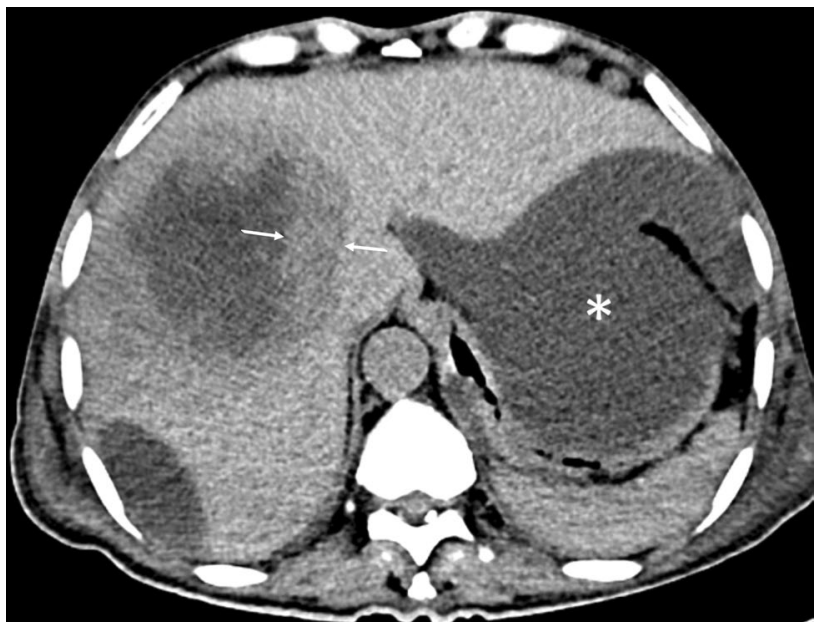

Fig. 7 Non-contrast CT image of a ruptured type I abscess in a 46-year old man showing an intermediate density zone (denoted by the two arrows), representing solid necrotic tissue. Note the localized perihepatic fluid collections resulted from the abscess rupture (asterisk)

intensive care unit; one with cecal perforation underwent surgery; two died from multiple organ failure.

The patients with type II abscesses had moderate symptoms; all had mild to moderate tenderness in the right upper quadrant and 12 (39\%) had intermittent fever. 19 (61\%) patients had completed antiamebic therapy before the CT. 15 of 31 patients $(48 \%)$ of the patients were successfully treated by antiamebic therapy alone and the remaining $52 \%$ required additional percutaneous drainage.

The patients with type III abscesses had mild tenderness in the right upper quadrant. All patients with type III abscesses had completed antiamebic therapy at home. Most of their symptoms had resolved by the time of examination, except for mild right upper quadrant pain. Their pain was due to the stretching effect exerted by the underlying large residual cavity on the liver capsule. Because all the patients were symptomatic, they required percutaneous drainage, even though the laboratory tests were normal.

\section{Discussion}

We identified three distinct morphological patterns of ALA - type I, II and III-with variable clinical and laboratory findings. Clinically, type I abscesses presented acutely with severe disease, whereas the other types presented with mild to moderate disease. Most type I abscesses responded poorly to antiamebic therapy and consequently required percutaneous drainage.

Type I abscesses - characterized by an absent or incomplete wall, ragged edges, multiple septa and interrupted or no enhancement-were observed in $66 \%$ of our cases. 

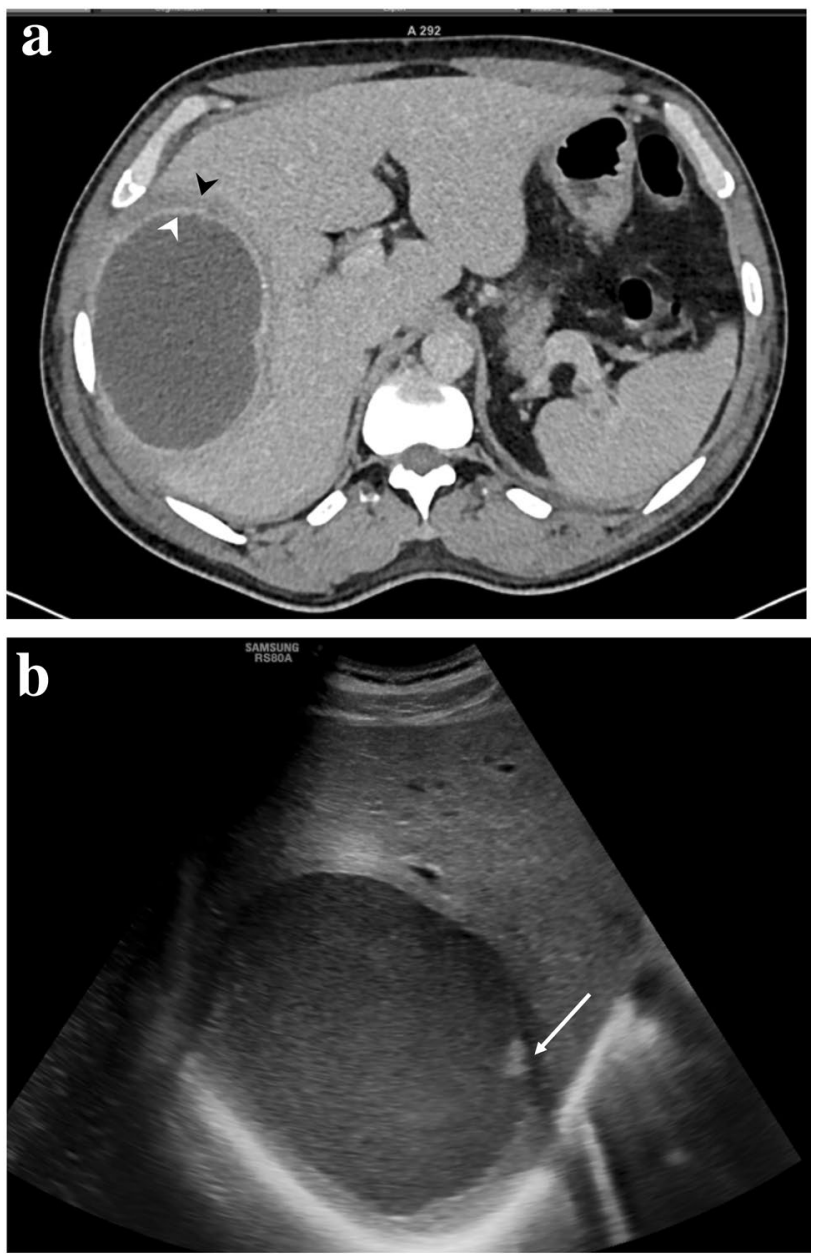

Fig. 8 a Axial CT image of a type II abscess in a 42-year-old man displaying double-target sign consisting of an inner enhancing ring (white arrowhead) and outer hypodense ring (black arrowhead). Clinically, he had intermittent fever and mild tenderness, but normal laboratory findings. His symptoms resolved immediately after needle aspiration of the abscess. b Sonogram of the abscess showing homogeneous fluid with minimal solid necrotic material (arrowhead)

This set of imaging findings suggests early abscess, usually observed in severe cases. Autopsy studies of fatal ALAs closely reflect this pattern [14, 21, 22]. Furthermore, although not viewed as the main features of ALAs, these findings appear to be specific. In their review on human amebiasis, Brandt and Tamayo et al. state that liver changes produced by amebae are quite characteristic and only rarely are they confused with lesions of a different nature, such as pyogenic abscesses or malignant neoplasms [21]. This pattern of ALA consists of liquefied necrotic tissue in the center and solid necrotic tissue adherent to the edges. Sonography is excellent in demonstrating the solid necrotic tissue, although the findings can sometimes be confused with hepatic malignant neoplasms [15]. The solid necrotic tissue can occasionally be

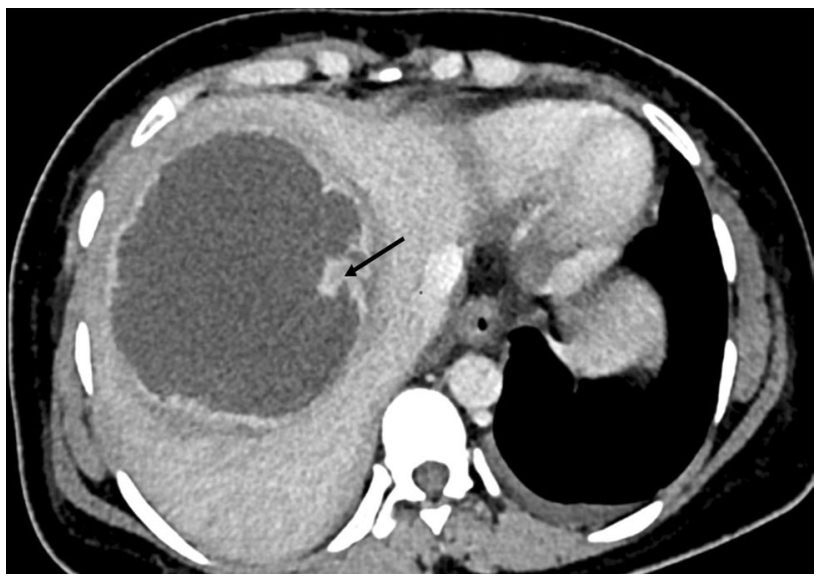

Fig. 9 CT image of a type II abscess in a 30-old-year old woman. Note the rim enhancing wall of the abscess and the perilesional halolike hypodensity. Also, note the focal nodularity in the wall (arrow) representing partially resorbed septa

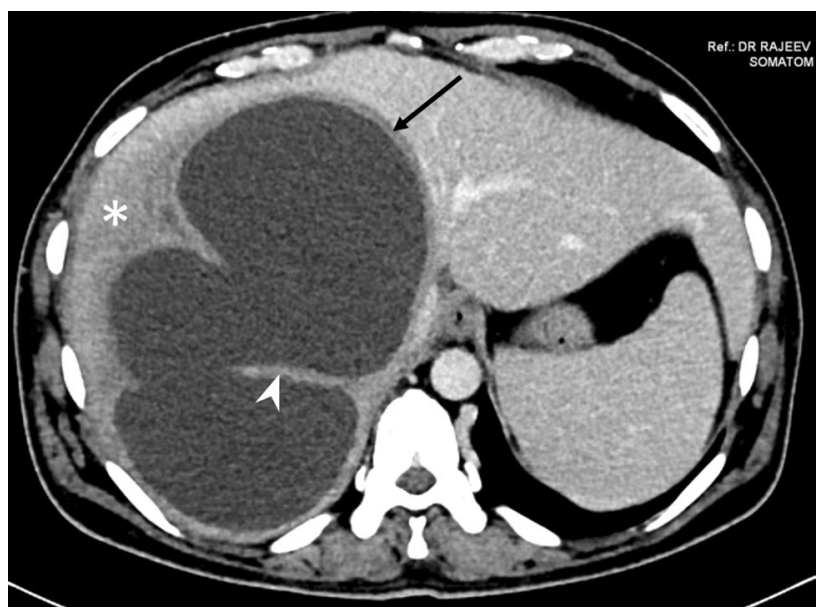

Fig. $10 \mathrm{CT}$ of a type III abscess in a 43-year-old man who presented with only mild tenderness in the right upper quadrant and had normal laboratory profile. The axial CT image shows the well-defined smooth wall (arrow), but without any contrast enhancement. There is an ill-defined hypodensity surrounding the abscess wall (asterisk). Note the large cavity formed by coalescence of multiple abscesses, and the intervening wall mimicking internal septations

identified by non-contrast CT as an intermediate density zone, but usually not by contrast-enhanced CT [10, 15].

The septa are known to be present in about one-third of ALAs on CT examinations, but their importance has not been recognized [10]. The septa of amebic abscesses are the cords of connective tissue which resists initial necrosis, but is subsequently resorbed as healing progresses [22]. Its presence therefore can indicate either an early stage of abscess or a poor response to medical treatment. Furthermore, the characteristics of septa can be considered distinctive of ALAs: they always arise from the inner edges of the abscesses and 


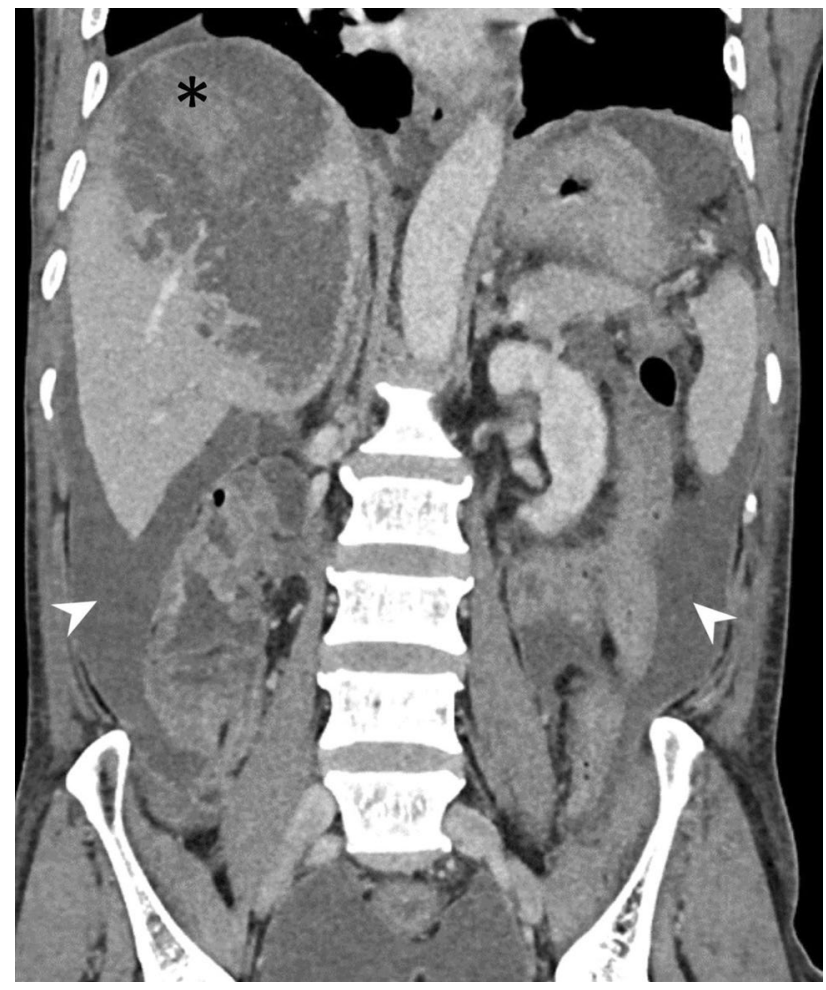

Fig. 11 CT of a 50-year-old man who presented acutely with severe pain and shock-like state. The coronal image demonstrates hyperdensity within the type I abscess cavity indicating the presence of hemorrhage (asterisk). The elliptical shape and polycyclic borders of the abscess are due to coalescence of two lesions. Also, note the diffuse intraperitoneal fluid collections (arrowheads) resulting from the abscess rupture

do not divide the cavity into multiple locules. In other words, ALAs are unilocular. Sometimes, however, coalescence of multiple abscesses may falsely give the impression of multilocular cavity, and the intervening parenchyma can be interpreted as internal septations. This might be the reason why the ALAs has occasionally been reported as multilocular [10]. This characteristic of the septa could be a useful finding for differentiating ALAs from malignant neoplasms, which contain internal septations [12].

The edges of type I abscesses showed irregular, interrupted or no enhancement. This observation might be a reflection of an interesting underlying pathologic phenomenon: amebic abscess has a scant inflammatory reaction at the edges in early phase, possibly because the amebic trophozoites at the edges cause lysis of the leukocytes, which in turn release mediators that cause extensive parenchymal necrosis $[4,21,23,24]$. As the ameba are killed by amebicidal drugs, inflammatory response is mounted at the edges to wall-off the necrotic process resulting in initial irregular and interrupted enhancement followed later by complete rim enhancement.
Type II abscesses are characterized by a complete rim enhancing wall. It must be emphasized that most reported CT imaging features in literature primarily concern type II abscesses. This pattern indicates healing phase and is observed in drug responsive disease [13]. Frequently, two rings (double-target sign) are identified-inner ring of enhancement, and outer ring of hypodensity. The inner ring is due to inflammatory hyperemia that lines the inner surface of the abscess wall, and the outer ring is due to edema [9, 13]. At this stage, the edema is confined only around the abscess wall, forming a hypodense halo; this type is unlike type I abscess where the edema is more widespread. Type II pattern is non-specific, sharing its morphological features with pyogenic abscesses [11].

Type III abscesses occur in the setting of treated abscess where the abscess content fails to resorb completely. Sonographic studies have identified the type III abscess as a round thick-walled cystic lesion with homogenous content. For this reason, it can sometimes be confused with benign hepatic cysts. In this regard, the CT findings obtained in this studya well-defined wall without enhancement and an ill-defined perilesional hypodensity_may be useful for diagnosis. Type III abscesses are clinically and biochemically inactive and therefore generally do not require treatment [16-18]. However, when sufficiently large to stretch the liver capsule, they can be painful to warrant percutaneous drainage; for this reason, all patients with type III abscesses in this study required drainage.

As shown in the results (Table 2), the CT-based morphological types paralleled clinical severity. The type I abscesses presented with severe clinical and biochemical findings. Most patients with this pattern required immediate percutaneous drainage and two died. Several additional CT findings of type I abscesses indicated increased disease severity: multiplicity of lesions, large size, local complications (rupture, intrahepatic duct dilatation, intracavitary hemorrhage or gas) — and remote complications (peritonitis, amebic colitis, appendicular or cecal perforation, ascites and pleural effusion). In addition, the laboratory results differed significantly from the other types, including leukocytosis, hyperbilirubinemia, hypoalbuminemia, elevated liver enzymes. Such parameters are known to be associated with a more acute and aggressive presentation [5-7, 20, 25, 26]. Large size and multiplicity of the lesions are the two most important factors that can be assumed to have caused the increased severity of disease in this type. The third factor may be the high prevalence of diffuse or wedge-shaped hypodensity in the type I abscesses, indicating widespread edema or ischemia $[9,19]$. In previous work, we have shown that the wedge-shaped hypoattenuating zone caused by portal or hepatic vein thrombosis are common in severe ALAs [19]. 
Table 2 Additional CT findings, clinical and laboratory findings, and treatment outcomes of the three morphological types of amebic liver abscesses

\begin{tabular}{|c|c|c|c|c|}
\hline Variables & $\begin{array}{l}\text { Type I } \\
(n=74)\end{array}$ & $\begin{array}{l}\text { Type II } \\
(n=31)\end{array}$ & $\begin{array}{l}\text { Type III } \\
(n=7)\end{array}$ & $p$-value \\
\hline \multicolumn{5}{|l|}{ Additional CT findings } \\
\hline Size of abscess $(\mathrm{cm})$ & $10.1 \pm 2.5$ & $8.6 \pm 1.4$ & $9.7 \pm 1.9$ & 0.010 \\
\hline Number of abscesses & $2.4 \pm 2.4$ & $1.4 \pm 0.9$ & $1.1 \pm 0.4$ & 0.039 \\
\hline Intracavitary hemorrhage & $4(5 \%)$ & $1(3 \%)$ & 0 & 0.744 \\
\hline Intrahepatic ductal dilations & $2(3 \%)$ & 0 & 0 & 0.593 \\
\hline Intracavitary air & $3(4 \%)$ & 0 & 0 & 0.453 \\
\hline Rupture & $42(57 \%)$ & $2(6 \%)$ & 0 & $<0.001$ \\
\hline Ascites & $23(31 \%)$ & $2(6 \%)$ & 0 & 0.007 \\
\hline Colonic manifestations & $28(38 \%)$ & $4(13 \%)$ & 0 & 0.008 \\
\hline Pleural effusion & $42(57 \%)$ & $4(13 \%)$ & 0 & $<0.001$ \\
\hline \multicolumn{5}{|l|}{ Clinical findings } \\
\hline Age (years) & $44.5 \pm 13.4$ & $41.1 \pm 9.1$ & $39.1 \pm 5.6$ & 0.277 \\
\hline Duration of symptoms (days) & $10.5 \pm 4.4$ & $27.6 \pm 6.5$ & $41.5 \pm 5.2$ & $<0.001$ \\
\hline Abdominal pain & $74(100 \%)$ & $31(100 \%)$ & $7(100 \%)$ & \\
\hline Fever & $62(83 \%)$ & $12(39 \%)$ & 0 & $<0.001$ \\
\hline \multicolumn{5}{|l|}{ Laboratory findings } \\
\hline $\mathrm{TLC}(/ \mu \mathrm{L})$ & $24,996 \pm 9,743$ & $12,620 \pm 4,826$ & $8,325 \pm 2,637$ & $<0.001$ \\
\hline Total bilirubin (mg/dL) & $2.4 \pm 1.2$ & $1.17 \pm 0.59$ & $0.54 \pm 0.22$ & $<0.001$ \\
\hline Serum albumin (g/dL) & $1.96 \pm 0.48$ & $2.74 \pm 0.56$ & $2.43 \pm 0.37$ & $<0.001$ \\
\hline AST (U/L) & $153.1 \pm 125.6$ & $54.6 \pm 28.4$ & $37.1 \pm 18.4$ & $<0.001$ \\
\hline ALT (U/L) & $123.7 \pm 118.9$ & $52.9 \pm 21.1$ & $31.4 \pm 5.2$ & 0.004 \\
\hline $\mathrm{ALP}(\mathrm{U} / \mathrm{L})$ & $343.6 \pm 146.01$ & $296.3 \pm 110.7$ & $254.7 \pm 59.5$ & 0.554 \\
\hline \multicolumn{5}{|l|}{ Treatment and outcomes } \\
\hline Inpatient hospital admission & $67(90.5 \%)$ & $11(35.5 \%)$ & $1(14.3 \%)$ & $<0.001$ \\
\hline Intensive care unit admission & $15(20.3 \%)$ & 0 & 0 & $<0.012$ \\
\hline Length of hospital stay (days) & $10.7 \pm 3.44$ & $6.1 \pm 1.9$ & 3 & $<0.001$ \\
\hline Drug alone & $14(19 \%)$ & $15(48 \%)$ & 0 & 0.002 \\
\hline Drainage & $60(81 \%)$ & $16(52 \%)$ & $7(100 \%)$ & 0.002 \\
\hline Surgery & $1(1 \%)$ & 0 & 0 & 0.772 \\
\hline Mortality & $2(3 \%)$ & 0 & 0 & 0.593 \\
\hline
\end{tabular}

$\mathrm{P}$ values in bold indicate statistically significant difference

Data are presented as $n(\%)$ or mean \pm standard deviation

$T L C$ total leukocyte counts, $A L T$ alanine aminotransferase, $A S T$ aspartate aminotransferase, $A L P$ alkaline phosphatase
In this study, type I abscesses presented acutely, whereas type II or type III had delayed presentation. Katzenstein et al. [25] emphasized the need to distinguish acute abscesses (those presenting within 10 days of onset of symptoms) from chronic. They found that over half of the patients with acute abscess, designated as acute aggressive type, had severe clinical symptoms and laboratory profile; they frequently required surgery; multiple abscesses were common in this group. In our previous study on ruptured ALAs, the median duration of symptoms before presentation was 10 days. In another study investigating the prognostic markers in ALAs, Sharma et al. [26] found that 24 of 135 patients died during the acute phase of disease. Thus, there is clear evidence that acute presentation is associated with more severe disease.

Rupture is the most common fatal complication of ALA. The two well established risk factors include large size and left lobe location; drainage has been advocated in presence of these factors, fearing that they will rupture [20, 27]. Also, the thickness $(<1 \mathrm{~cm})$ of liver tissue between abscess and liver margin has reportedly been used as an objective measure to assess impending rupture $[2,26,28]$. In this context, our results strongly point out that lack of a mature wall (type I abscesses) on CT is perhaps the single most important risk factor that must be considered while assessing rupture risk. Most ruptures were observed when the abscesses lacked a mature wall. 


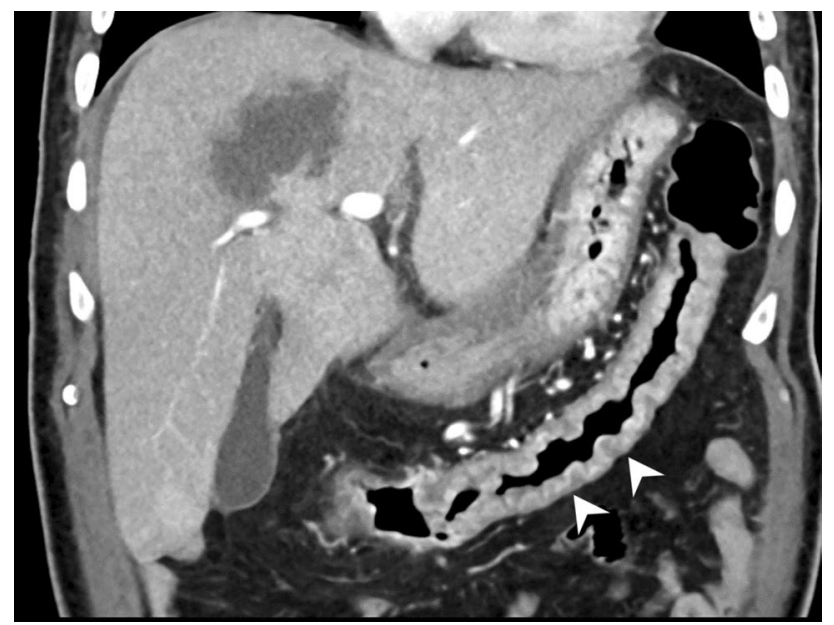

Fig. 12 Amebic colitis in a 62-year-old-man with type I amebic liver abscess. The coronal CT image shows a symmetrically thickened and enhancing wall of the transverse colon (arrowheads)

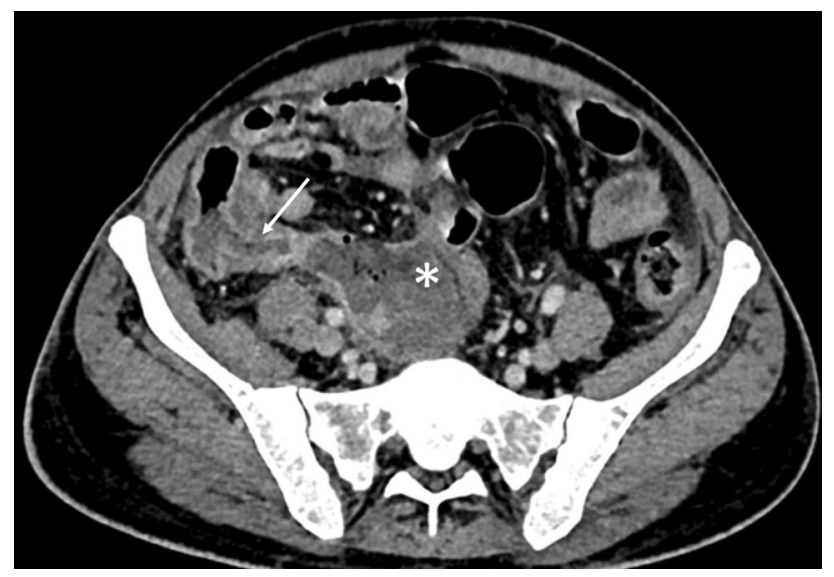

Fig. 13 Axial CT image of a 62-year-old man obtained at the level of the root of the appendix in this patient showing the cecal wall thickening, ruptured appendix (arrow) and subsequent localized fluid collection in the pelvis (asterisk)

The incidence of colitis was high in type I abscesses compared with the others. This observation is similar to a recent Indian study that found concurrent colitis more common with severe disease [29]. A high prevalence of colitis (over $60 \%$ ) in autopsy studies further confirms this view [30]. We observed that CT findings of amebic colitis were similar to that of inflammatory bowel disease, but the occurrence of cecal or appendicular perforation in 4 of 24 patients of type I abscesses with concurrent colitis is noteworthy.

Based on the sonographic criteria somewhat similar to the CT criteria adopted in this paper, N'Gbesso could also classify the ALAs into three types [17]. Most of their type I abscesses responded to medical treatment alone; type II required drainage; and type III required no treatment.
However, we observed conflicting results (Table 2). This discrepancy may be explained by several notable differences in the patient population and the methodology. First, the previous study classified the abscesses based on the initial sonographic findings (before initiation of the medical treatment), and the outcomes were assessed prospectively. This study classified ALAs based on CT features, and all involved patients had received medical treatment, either partial or complete, before the examination; this might have influenced the morphological patterns. Second, the previous studies had smaller type I abscesses (<less than $10 \mathrm{~cm}$ ) [17]. Most patients in our study were referred from gastroenterology clinics for percutaneous drainage. Their abscesses were large and multiple. This led to a clear selection bias toward severe cases. Last, because this study included only symptomatic cases, the type III abscesses (typically asymptomatic residual cavity and do not warrant any therapy) required drainage. Therefore, the treatment outcomes observed in this study can be misinterpreted, if not viewed in light of these limitations.

To conclude, ALAs can present with three different morphological types. The type I pattern is associated with severe clinical symptoms and deranged laboratory parameters, and higher incidence of local and remote complications. This pattern often has poor response to medical therapy and therefore requires percutaneous drainage.

Funding No funding was received for this study.

\section{Declarations}

Conflict of interest All authors declare that they have no conflict of interest.

Ethical approval All procedures performed in studies involving human participants were in accordance with the ethical standards of the institutional and/or national research committee and with the 1964 Helsinki declaration and its later amendments or comparable ethical standards.

Informed consent Our institutional review board approved this study and informed consent was obtained from all patients.

\section{References}

1. Zibari GB, Maguire S, Aultman DF, McMillan RW, McDonald JC (2000) Pyogenic liver abscess. Surg Infect (Larchmt) 1:15-21. https://doi.org/10.1089/109629600321254.

2. Ghosh S, Sharma S, Gadpayle AK, Gupta HK, Mahajan RK, Sahoo R, Kumar N (2014) Clinical, laboratory, and management profile in patients of liver abscess from northern India. J Trop Med 2014:142382. https://doi.org/10.1155/2014/142382.

3. Sharma N, Sharma A, Varma S, Lal A, Singh V (2010) Amoebic liver abscess in the medical emergency of a North Indian hospital. BMC Res Notes 3:21. https://doi.org/10.1186/1756-0500-3-21. 
4. Stanley SL Jr (2003) Amoebiasis. Lancet 361:1025-34. https:// doi.org/10.1016/S0140-6736(03)12830-9.

5. Chuah SK, Chang-Chien CS, Sheen IS, Lin HH, Chiou SS, Chiu CT, Kuo CH, Chen JJ, Chiu KW (1992) The prognostic factors of severe amebic liver abscess: a retrospective study of 125 cases. Am J Trop Med Hyg 46:398-402. https://doi.org/10.4269/ajtmh. 1992.46.398.

6. Priyadarshi RN, Prakash V, Anand U, Kumar P, Jha AK, Kumar R (2019) Ultrasound-guided percutaneous catheter drainage of various types of ruptured amebic liver abscess: a report of 117 cases from a highly endemic zone of India. Abdom Radiol (NY) 44:877-885. https://doi.org/10.1007/s00261-018-1810-y.

7. Sánchez-Aguilar M, Morán-Mendoza $O$, Herrera-Hernández MF, Hernández-Sierra JF, Mandeville PB, Tapia-Pérez JH, et al (2012) Prognostic indications of the failure to treat amoebic liver abscesses. Pathog Glob Health 106:232-7. https://doi.org/10.1179/ 2047773212 Y.0000000021.

8. Jha AK, Jha P, Chaudhary M, Purkayastha S, Jha SK, Ranjan R, Priyadarshi RN, Kumar R (2019) Evaluation of factors associated with complications in amoebic liver abscess in a predominantly toddy-drinking population: A retrospective study of 198 cases. JGH Open 3:474-479. https://doi.org/10.1002/jgh3.12183.

9. Mathieu D, Vasile N, Fagniez PL, Segui S, Grably D, Lardé D (1985) Dynamic CT features of hepatic abscesses. Radiology 154:749-52. https://doi.org/10.1148/radiology.154.3.3969480.

10. Radin DR, Ralls PW, Colletti PM, Halls JM (1988) CT of amebic liver abscess. AJR Am J Roentgenol 150:1297-301. https://doi. org/10.2214/ajr.150.6.1297.

11. Halvorsen RA, Korobkin M, Foster WL, Silverman PM, Thompson WM (1984) The variable CT appearance of hepatic abscesses. AJR Am J Roentgenol 142:941-6. https://doi.org/10.2214/ajr. 142.5.941.

12. Qian LJ, Zhu J, Zhuang ZG, Xia Q, Liu Q, Xu JR (2013) Spectrum of multilocular cystic hepatic lesions: CT and MR imaging findings with pathologic correlation. Radiographics 33:1419-33. https://doi.org/10.1148/rg.335125063.

13. Elizondo G, Weissleder R, Stark DD, Todd LE, Compton C, Wittenberg J, Ferrucci JT Jr (1987) Amebic liver abscess: diagnosis and treatment evaluation with MR imaging. Radiology 165:795800. https://doi.org/10.1148/radiology.165.3.2891154.

14. Simjee AE, Patel A, Gathiram V, Engelbrecht HE, Singh K, Rooknoodeen $F(1985)$ Serial ultrasound in amoebic liver abscess. Clin Radiol 36:61-8. https://doi.org/10.1016/s0009-9260(85)80027-1.

15. Terrier F, Becker CD, Triller JK (1983) Morphologic aspects of hepatic abscesses at computed tomography and ultrasound. Acta Radiol Diagn (Stockh) 24:129-37. https://doi.org/10.1177/02841 8518302400205 .

16. Ralls PW, Quinn MF, Boswell WD Jr, Colletti PM, Radin DR, Halls J (1983) Patterns of resolution in successfully treated hepatic amebic abscess: sonographic evaluation. Radiology 149:541-3. https://doi.org/10.1148/radiology.149.2.6622702.

17. N'Gbesso RD, Kéita AK. Echographie des abcès amibiens du foie (1997) Proposition d'une nouvelle classification [Ultrasonography of amebic liver abscesses. Proposal of a new classification]. J Radiol 78:569-76. French.
18. Nari GA, Ceballos Espinosa R, Carrera Ladrón de Guevara S, Preciado Vargas J, Cruz Valenciano JL, et al (2008) Tres años de experiencia [Amebic liver abscess. Three years experience]. Rev Esp Enferm Dig 100:268-72. Spanish. https://doi.org/10.4321/ s1130-01082008000500004

19. Priyadarshi RN, Kumar P, Kumar R, Anand U, Shyama (2020) Venous thrombosis and segmental hypoperfusion in amebic liver abscess: MDCT demonstration and its implications. Abdom Radiol (NY) 45:652-660. https://doi.org/10.1007/ s00261-020-02409-6.

20. Hughes MA, Petri WA Jr (2000) Amebic liver abscess. Infect Dis Clin North Am.14:565-82, viii. https://doi.org/10.1016/s08915520(05)70121-5.

21. Brandt H, Tamayo RP (1970) Pathology of human amebiasis. Hum Pathol 1:351-85. https://doi.org/10.1016/s0046-8177(70) 80072-7.

22. Jimenez F (1981) Pathology of amebiasis. Bull N Y Acad Med 57:217-23

23. Mortelé KJ, Segatto E, Ros PR (2004) The infected liver: radiologic-pathologic correlation. Radiographics 24:937-55. https:// doi.org/10.1148/rg.244035719.

24. Tsutsumi V, Martinez-Palomo A (1988) Inflammatory reaction in experimental hepatic amebiasis. An ultrastructural study. Am J Pathol. 130:112-9.

25. Katzenstein D, Rickerson V, Braude A. (1982) New concepts of amebic liver abscess derived from hepatic imaging, serodiagnosis, and hepatic enzymes in 67 consecutive cases in San Diego. Medicine (Baltimore) 61:237-46. https://doi.org/10.1097/00005 792-198207000-00003.

26. Sharma MP, Dasarathy S, Verma N, Saksena S, Shukla DK (1996) Prognostic markers in amebic liver abscess: a prospective study. Am J Gastroenterol 91:2584-8.

27. Kurland JE, Brann OS (2004) Pyogenic and amebic liver abscesses. Curr Gastroenterol Rep 6:273-9. https://doi.org/10. 1007/s11894-004-0078-2.

28. Goyal A, Dhaliwal HS, Nampoothiri RV, Singh R, Abraham J, Sharma R, et al (2021) Percutaneous catheter drainage of uncomplicated amoebic liver abscess: prospective evaluation of a clinical protocol for catheter removal and the significance of residual collections. Abdom Radiol (NY) https://doi.org/10.1007/ s00261-021-02949-5.

29. Premkumar M, Devurgowda D, Dudha S, Kulkarni A, Joshi YK (2019) Clinical and Endoscopic Management of Synchronous Amoebic Liver Abscess and Bleeding Colonic Ulcers. J Assoc Physicians India 67:14-18.

30. Aikat BK, Bhusnurmath SR, Pal AK, Chhuttani PN, Datta DV (1979) The pathology and pathogenesis of fatal hepatic amoebiasis--A study based on 79 autopsy cases. Trans R Soc Trop Med Hyg 73:188-92. https://doi.org/10.1016/0035-9203(79)90209-8.

Publisher's Note Springer Nature remains neutral with regard to jurisdictional claims in published maps and institutional affiliations. 